\title{
A new dynamic surface reconstruction method for cardiac 3D mapping procedure
}

\author{
Yingsong Huang ${ }^{1, a}$, Jian $\mathrm{Wu}^{1, \mathrm{~b}}$, Riqing $\mathrm{Chen}^{1, \mathrm{c}}$ \\ ${ }^{1}$ Research Center of Biomedical Engineering Graduate School of Shenzhen, Tsinghua University, \\ Shenzhen, China, 518055 \\ aemail: yingsong_huang@163.com, bemail:wuj@sz.tsinghua.edu.cn, \\ cemail:chenrq12@mails.tsinghua.edu.cn
}

\begin{abstract}
Keywords: Cardiac Mapping; Dynamic Surface Reconstruction; Delaunay Based; Iterative Replacement
\end{abstract}

\begin{abstract}
During cardiac 3D mapping procedure, the dynamic surface reconstruction of heart chambers helps to diagnose cardiac disease and improve the operation precision by keeping the catheter in place. The paper introduces a new fast dynamic surface reconstruction method considering the distribution characteristics of the sample points collected during ablation. The algorithm defines the possible optimum reconstructed surface embedding in the Delaunay diagram of the sample points, and uses an iteration method to construct the defined optimum surface. The optimum reconstructed surface constraints can guarantee that the reconstructed surface is geometrically approximate to the original surface. The algorithm successively inserts points into the reconstructed surface by replacing the surface structure in a small neighbor of the inserted points. In order to keeping the integrity of the surface, the algorithm completes the replacement on basis of inside/outside of the Delaunay diagram. In order to assure a topological equivalence between the reconstructed surface and the original surface, the paper introduces an inflating method to keep reconstructed surface manifold. The reconstructed surface is smoothed by the Loop subdivision algorithm. The paper experiments on six left atrium (LA) CT models, and the reconstruction results demonstrate that the proposed method can progressively reconstruct a surface which is geometrically and topologically approximate to the original heart chamber with a fast speed.
\end{abstract}

\section{Introduction}

Cardiac 3D mapping systems play an important role in the treatment of arrhythmia, and 3D visualization of heart chambers is a critical part of these systems. During the cardiac mapping procedure, an interventionalist manually collects points from endocardium with a mapping catheter and the endocardium surface model is progressively reconstructed with these scatter points. These unorganized points are acquired one by one until the reconstructed surface meets the mapping requirement. The points cloud used for reconstruction may be sparse and unevenly distributed due to random acquisition. Moreover, the surface reconstruction has to be efficient enough to follow the speed of points acquisition.

Most of current surface reconstruction methods fall into three categories: methods based on implicit function, methods based on geometric deformation, and methods based on Delaunay/Voronoi diagram. Hoppe ${ }^{[1]}$ firstly proposes the early implicit function based method. The method estimates points normal, and normalizes the normals by use of a neighborhood graph. The method considers surface reconstruction as a procedure solving zero-level set of an implicit signed distance function and extracting surface facets with marching cube ${ }^{[2]}$. The method proposed by Kazhdan $^{[3]}$ reconstructing surface by solving poisson equations is an improvement of it. Power crust $^{[4]}$ provides guarantees that the surface reconstructed with the proposed method based on Delaunay is geometrically and topologically approximate to the original surface under the condition of $\varepsilon$-sample. Peethambaran $\mathrm{J}$ et al. ${ }^{[5]}$ introduce the concept of divergent concavity, and accordingly define a proximity graph called as shape-hull graph(SHG(S)). They propose a simple sculpture method on basis of the Delaunay diagram to construct the defined $S H G(S)$ and take the boundary of $S H G(S)$ as the reconstructed surface. The algorithm requires sufficiently dense sampling as well. 
Reisfeld $^{[6]}$ proposes a geometric deformation method based on radial distance to reconstruct intra-body organs. The method firstly establishes an initial closed mesh, and uses the mesh to interpolate sample points with a first deformation and subsequent fine adjustment. Chiang ${ }^{[7]}$ improves the Reisfeld method, and avoids the high distortion for small number of contact points. Moreover, the method resolves the problem of non-convergence in the smoothing process by introducing the local Loop subdivision ${ }^{[8]}$.

Most of these methods is aimed at one-off surface reconstruction, and cannot apply to dynamic surface reconstruction. The implicit function based methods and the geometric deformation methods have a higher computational complexity compared to the Delaunay based methods. Though the Chiang method can progressively reconstruct surfaces, its efficiency can hardly meet the real time requirement, especially for the improvement of the electromagnetic localization technology.

The paper proposes a dynamic surface reconstruction method based on Delaunay diagram. The method fits in sparsely and unevenly sampling condition and has a high iteration speed compared to prior art.

\section{The optimum reconstructed surface}

Assume that there is a surface $S \in \mathrm{R}^{3}$, a points set $P \in S$, and a reconstructed surface $\Gamma$, which is a polygon composed of triangles interpolates $P . \Gamma$ is 2-manifold only if each vertex on it is regular. A vertex is regular if it has a surface neighbor which is homeomorphic to a disk, otherwise the vertex is singular. ${ }^{[9]}$ If a vertex is regular, it forms the center of exactly one closed triangle fan, which we name an umbrella of the vertex. Take $D T(P)$ as the Delaunay diagram of $P$, and we have $\Gamma \subseteq D T(P)$ in this paper. $\Gamma$ partitions $D T(P)$ into two parts, the tetrahedron set outside $\Gamma T_{o}$ and the tetrahedron set inside $\Gamma \quad T_{i}$, and $\Gamma$ is the border $\delta T_{i}$. Take $P_{k}=\left\{p_{0}, p_{1}, \cdots, p_{k-1}\right\}$ as the first $k$ points used in reconstruction, and the reconstructed surface at iteration step $k$ is marked as $\Gamma_{k} \subseteq D T\left(P_{k}\right)$.

Definition 1. The distance from the surface $\Gamma$ to the points set $P$ is defined as equation $1 .^{[10]}$ Accordingly, we define the distance from the point set $P$ to the surface $\Gamma$ as equation 2 .

$$
\begin{gathered}
E(\Gamma)=\left(\int_{x \in \Gamma} d_{\Gamma}{ }^{t}(x) d \phi\right)^{1 / t}, 1 \leq t \leq \infty \\
E^{\prime}(\Gamma)=\left(\sum_{p \in P} d_{p}{ }^{t}(x)\right)^{1 / t}, 1 \leq t \leq \infty
\end{gathered}
$$

Where $d_{\Gamma}(x)$ is the distance from $x \in \Gamma$ to its closest point in $P$, and $d_{p}(x)$ is the distance from $p \in P$ to its closest point $x$ on $\Gamma$.

Definition 2. Mark $\Gamma$ as the set of all the possible 2-manifold polygons interpolating $P$, and the optimum reconstructed surface with $P$ is defined as the inclusive polygon that minimizes $E^{\prime}(\Gamma)$ and $E(\Gamma)$ simultaneously, mathematically as equation 3.

$$
\Gamma_{\text {opt }}=\arg \min _{\Gamma \in \Gamma}\left\{E^{\prime}(\Gamma), E(\Gamma)\right\}
$$

In the paper, we have $\Gamma \subseteq D T(P)$.

\section{Updating reconstructed surface by local facets replacement}

Assume that we have already extracted the optimum reconstructed surface of the first $k$ points $\Gamma_{k}$ from $D T\left(P_{k}\right)$ at step $k$, and we can insert the $(k+1)$ th point $p_{k}$ into surface to acquire $\Gamma_{k+1}$ without producing singularities by using an umbrella $U$ centered on $p_{k}$ to replace the part 
of $\Gamma_{k}$ enclosed by $U$ which is composed of some adjacent triangles.

When the point $p_{k}$ is inserted into the Delaunay diagram $\operatorname{DT}\left(P_{k}\right)$, some tetrahedra in neighbor of $p_{k}$ are removed to cause a polytope hole which will be filled in by new created tetrahedra to keep the new Delaunay diagram $D T\left(P_{k+1}\right)$ solid. Each of the new tetrahedra is composed of $p_{k}$ and a triangle on the boundary of the polytope hole. Therefore, if we select some adjacent triangles either embedded in the hole or on the boundary of the hole from $\Gamma_{k}$, and these triangles compose a regular simplicial 2-complex marked as $L$ with boundary edges on the boundary of the hole, each of these boundary edges will be an edge of a new created triangle incident to $p_{k}$, and these new created triangles will compose a umbrella centered on $p_{k}$ marked as $U$. If we replace $L$ with $U$, we will insert $p_{k}$ into the reconstructed surface and every vertex in $U$ is regular. Taking Fig.1 as an example, when we insert a point $p$ into $D T\left(P_{k}\right)$, adjacent surface facets $p_{0} \widehat{p_{1}} p_{2}, p_{0} \widehat{p_{2}} p_{3}$, and $\hat{p}_{0} \widehat{p}_{3} p_{4}$ each of which is either embedded in the polytope hole cause by $p$, or on its boundary, the set of them marked as $L$. Moreover, the boundary edges of $L \quad \widehat{p_{0} p_{1}}, \widehat{p_{1} p_{2}}, \widehat{p_{2} p_{3}}, \widehat{p_{3} p_{4}}$, and $\widehat{p_{4} p_{0}}$ are on the boundary of the hole, and the new created triangles $p_{0} \widehat{p}_{1} p, \hat{p}_{1} \widehat{p}_{2} p, p_{2} \widehat{p}_{3} p, \hat{p}_{3} \widehat{p}_{4} p$, and $\hat{p}_{4} \widehat{p}_{0} p$ which are composed of these boundary edges and $p$ compose a umbrella centered on $p$ marked as $U$. In our method, we use $U$ to replace $L$ to insert $p$ into the reconstructed surface. The whole replacement procedure in 2D is shown as Fig.2. In order to insert point $C$ into the reconstructed curve, we use an umbrella incident to $C$ composed of $\widehat{A C}$ and $\widehat{C B}$ to replace $\widehat{A B}$ which is more closer to $C$ than any other surface facets.

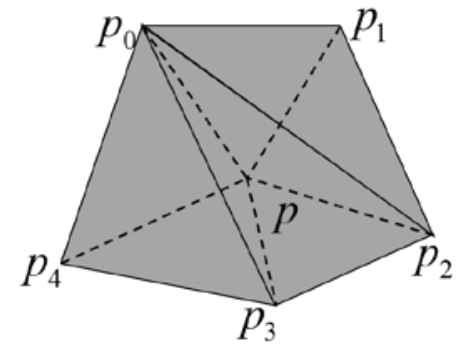

Fig. 1. The local replacement of the reconstructed surface

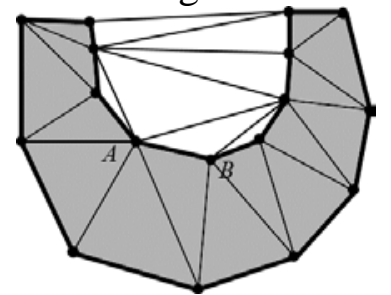

(a) Before $C$ inserted

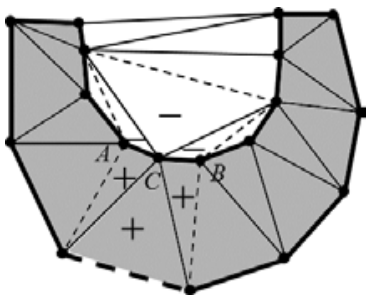

(b) After $C$ inserted

Fig. 2. The replacement procedure in $2 \mathrm{D}$

To avoid possible holes on the reconstructed surface created during replacement, the method completes surface replacement by means of inside or outside labeling of Delaunay tetrahedra. To keep the reconstructed surface manifold, the method inflates the surface in the neighbor of singularity. We mark the operation that remove a tetrahedron from $T_{i}$ into $T_{o}$ as sculpture, and mark the operation that add a tetrahedron in $T_{o}$ into $T_{i}$ as inflate. The sculpturing or inflating operation is actually tetrahedra labeling as well. 


\section{Constructing the optimum surface using an iteration method}

We introduce an iteration method to dynamic reconstruct the optimum surface defined before by local surface facets replacement. At each iteration step, we insert a sample point into the reconstructed surface by replacing some adjacent facets in a neighbor of it with an umbrella and thereafter some subsequent restoration may also be needed. We have to minimize the total increments of error produced during the replacement and the restoration procedure in order to acquire the optimum surface. The total error increments are defined as equation 4.

$$
\begin{aligned}
& \Delta E^{\prime}(\Gamma)=E^{\prime}\left(\Gamma_{k+1}\right)-E^{\prime}\left(\Gamma_{k}\right) \\
& \Delta E(\Gamma)=E\left(\Gamma_{k+1}\right)-E\left(\Gamma_{k}\right)
\end{aligned}
$$

Either the replacement or the restoration process is a series of surface facets replacement. In our method, the surface facets replacement is based on inside/outside labeling of Delaunay tetrahedra. So we only need to compute the error increments produced in tetrahedra labeling. In order to minimize $\Delta E^{\prime}(\Gamma)$, we only need to make sure that the reconstructed surface contains sample points as many as possible. Then the main problem is to minimize $\Delta E(\Gamma)$. Firstly, we define the error increments produced during sculpturing or inflating a tetrahedron $t$ while labeling. The definition is as equation 5 .

$$
\begin{aligned}
& \Delta E_{\text {inflate }}(t)=E\left(\delta\left(T_{i} \cup\{t\}\right)\right)-E\left(\delta\left(T_{i}\right)\right) \\
& \Delta E_{\text {shrink }}(t)=E\left(\delta\left(T_{i} \backslash t\right)\right)-E\left(\delta\left(T_{i}\right)\right)
\end{aligned}
$$

In order to keep the topologic property of the reconstructed surface, the number of vertices, edges, and facets adjacent to the surface on the tetrahedra sculptured or inflated have to be $(3,3,1)$, $(4,5,3)$, or $(4,6,3)$ as shown in Fig.3.

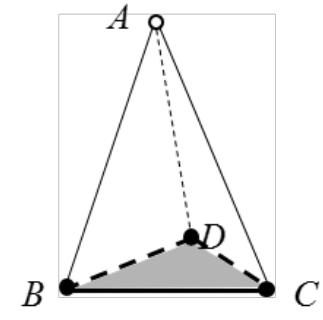

(a) $(3,3,1)$

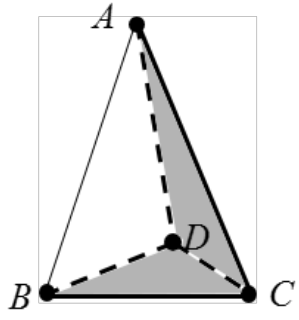

(b) $(4,5,3)$

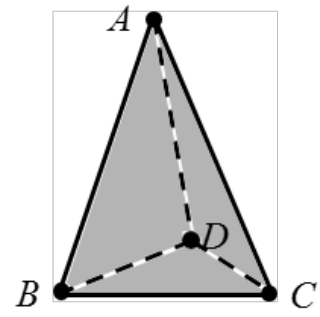

(c) $(4,6,3)$

Fig. 3 . The tetrahedra sculptured or inflated. The vertices, edges, and facets mark gray are on the reconstructed surface

Then we compute the error increments $\Delta E(t)$ produced by sculpturing or inflating a tetrahedron $t$. Firstly, considering (a) in Fig.3, when the tetrahedron is sculptured or inflated, the surface facet $B \widehat{C} D$ is replaced by the facets $A \widehat{B} C, \widehat{A C D}, A \widehat{D} B$ and the vertex $A$ is inserted into the reconstructed surface. We assume that the error increments $\Delta E<0$ and the value is proportional to its circumradius if the smallest circumscribing ball of the facet $\widehat{B C} D$ encloses the vertex $A$ according to the conclusion in Shrink ${ }^{[10]}$ and $S H G(S)^{[5]}$. Otherwise, we compute the distance from each facet to the sample points by use of the dual Voronoi diagram of the facet at first. As shown in Fig.4, we calculate the distance of each Voronoi cell in the facet, and sum them as the distance of the whole facet. The result is shown in equation 8 when 1-norm is chosen as the distance function. Then the error increments is calculated as 


$$
\Delta E(\triangle A B C D)=E(A B C)+E(A C D)+E(A D B)-E(B C D)
$$

Labeling the tetrahedron of (c) in Fig.3 is an inverse process of (a) with an isolate vertex $D$ are induced. The error increments produced in sculpturing or inflating the tetrahedron in (c) is

$$
\triangle E(\triangle A B C D)=E(B C D)-E(A B C)-E(A C D)-E(A D B)
$$

It is hard to directly calculate the error increments in labeling the tetrahedron of (b). However, $E^{\prime}(\Gamma)$ does not change when labeling it because none of its vertex is inserted into surface or become an isolate vertex. So we only need to estimate whether the error increments is greater than 0 . We assume that the error increments is less than 0 if the smallest circumscribing ball of either $\triangle A C D$ or $\triangle B C D$ encloses its opposite vertex and $|A B|<|C D|$, otherwise the error increments is greater than 0 .

$$
\begin{aligned}
& E(B C D)=E(B E O F)+E(C E O G)+E(D F O G) \\
& =\int_{x \in B E O F}\|x-B\|_{1} d x+\int_{x \in C E O G}\|x-C\|_{1} d x+\int_{x \in D F O G}\|x-D\|_{1} d x \\
& =\frac{1}{24}|B C|^{3}\left(\cot \angle D+\frac{1}{2} \cot ^{2} \angle D\right)+ \\
& \frac{1}{24}|C D|^{3}\left(\cot \angle B+\frac{1}{2} \cot ^{2} \angle B\right)+ \\
& \frac{1}{24}|B D|^{3}\left(\cot \angle C+\frac{1}{2} \cot ^{2} \angle C\right)
\end{aligned}
$$

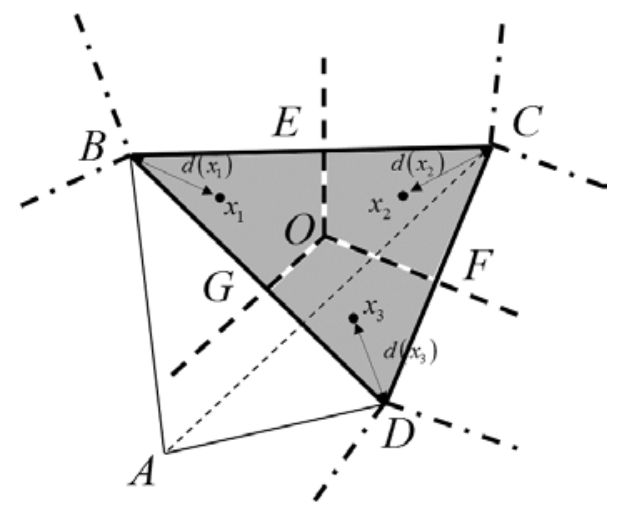

Fig. 4. The calculation of the error increments when sculpturing or inflating the tetrahedron $\mathrm{ABCD}$ with only one facet in the surface

Our method is a recursive iteration process, and every iteration step is accomplished by surface facets replacement based on tetrahedra labeling. We construct the optimum surface by minimizing the total error increments and reinserting isolates vertices at each iteration. Assume that the error increments produced when labeling a tetrahedron $t_{i}$ is marked as $\Delta E\left(t_{i}\right)$ and that the total error increments at the iteration is marked as $\Delta E(\Gamma)$, and then we have

$$
\Delta E(\Gamma)=\sum_{i}^{n} \Delta E\left(t_{i}\right)
$$

\section{Smoothing by Loop subdivision}

In order to smooth the surface mesh reconstructed by the iteration replacement algorithm, a global subdivision by Loop algorithm ${ }^{[8]}$ is applied to the mesh for its simplicity and effectiveness. 


\section{Test results}

In order to validate the effect of the proposed method, we implement the algorithm with $\mathrm{C}++$ on an Intel Pentium @2.8GHz processer system and experiment on reconstruction of six heart chamber models. These models are acquired from coronary CT angiography images scanned by PHILIPs Brilliance 64-slice spiral CT with manual segmentation and surface extraction using Marching Cube. In the experiments, we emulate the points collection process in a regular heart mapping procedure in AF surgery. Firstly, we select a group of circular points at the pulmonary vein branch to separate heart chamber models from pulmonary. Then we randomly acquire points from the heart chamber models one by one and progressively reconstruct the original models. As shown in Fig.5, our can progressively reconstruct the heart chamber models with good visual effects.

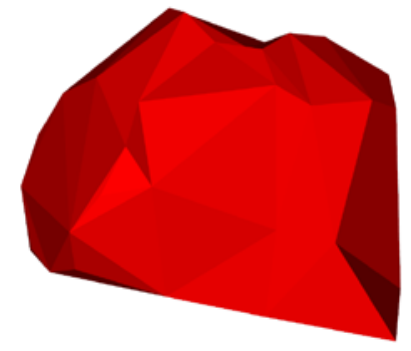

(a) Reconstructed surface with 50 sample points

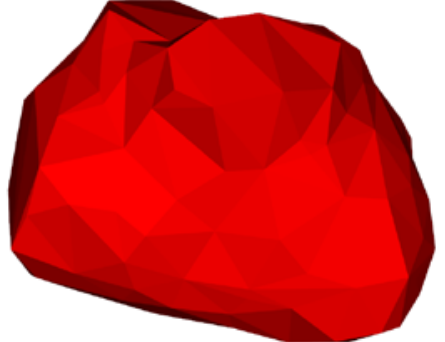

(b) Reconstructed surface with 150 sample points

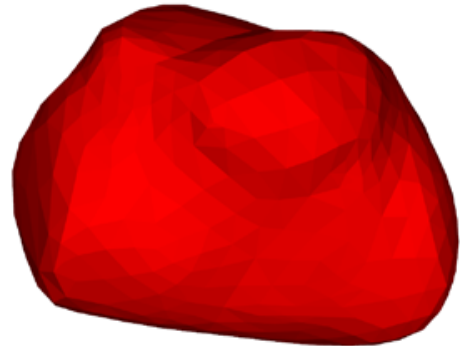

(c) Reconstructed surface after subdivision

Fig. 5. The progressive reconstruction of Left atrium

Fig.6 is the superimposed comparison between the reconstructed surface and the corresponding heart chamber models from CT. According to the comparison results, we can see the reconstructed surface is geometrically and topologically approximate to the original surface.

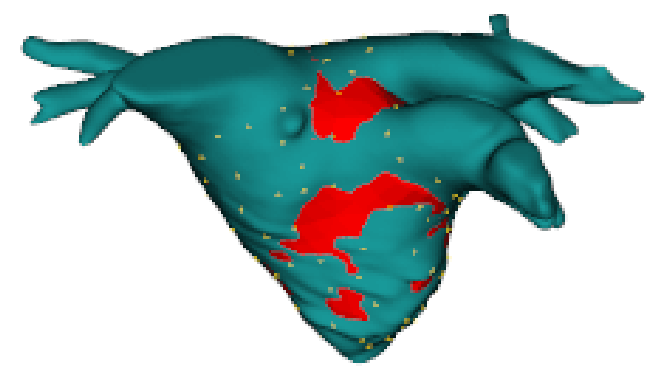

(a) LA1

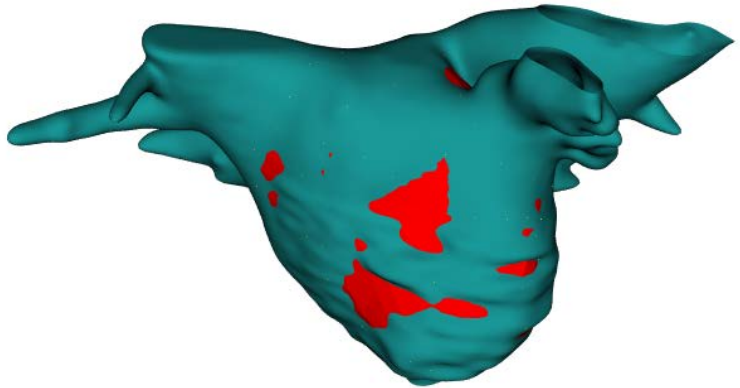

(c) LA3

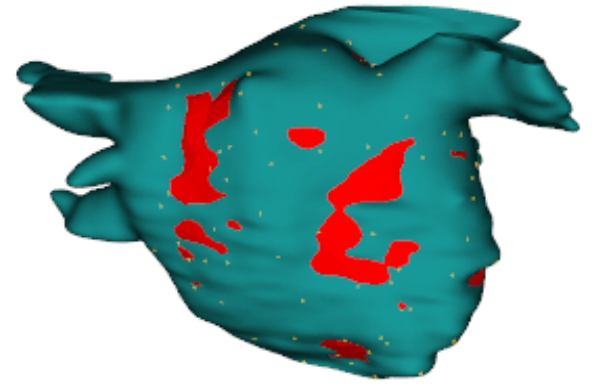

(b) LA2

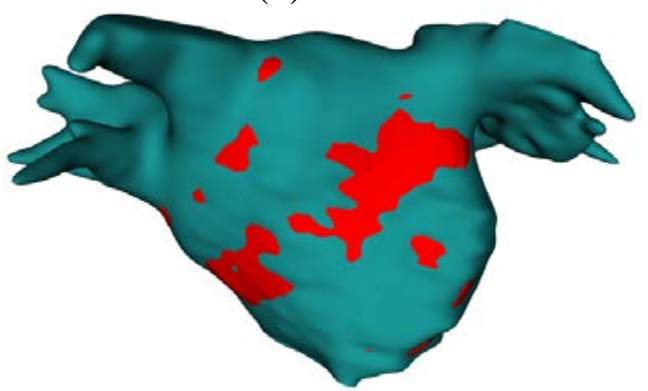

(d) LA4

Fig. 6. The superimposed comparison between four Left atrium models (marked blue) from CT and the corresponding reconstructed surfaces (marked red) by our method with 150 sample points

In order to estimate the change trend of error precision between the reconstructed surface and the original surface, we calculate the error of the reconstructed surface every 10 points inserted along with the progressive reconstruction of LA1. We estimate the distance of 100 points acquired from the original model to the reconstructed surface at each step as shown in Fig.7. The maximum error decreases to $1.1603 \mathrm{~mm}$ and the mean square error decreases to $0.5551 \mathrm{~mm}$ when the number of sample points comes to 100 . 


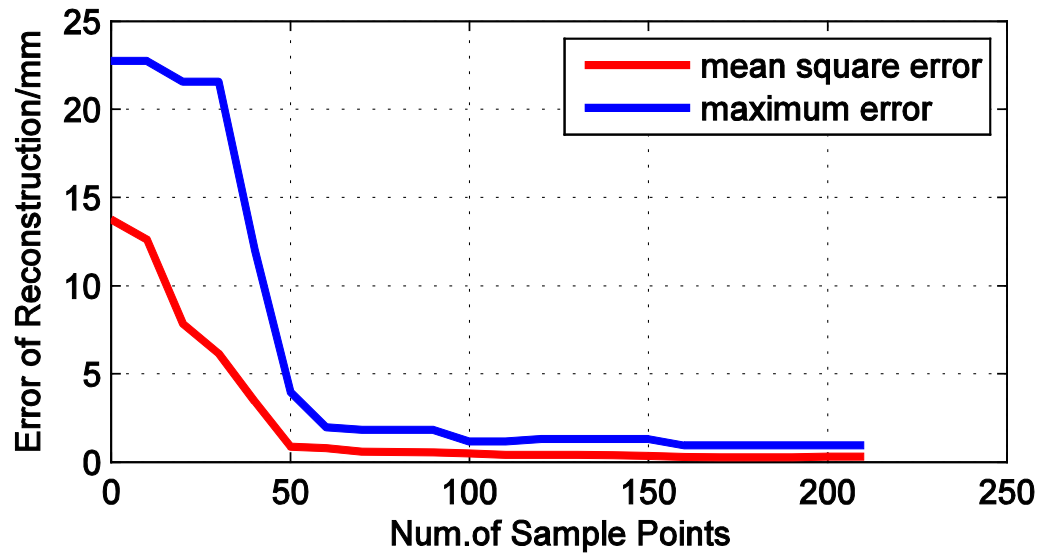

Fig. 7. Statistics of error precision

In addition, we estimate the consumed time at each iteration step by calculating the average of iteration time over 10 points. Fig. 8 is the statistics of consumed time at each reconstruction step of six Left atrium models. From the results, we can see the update time at each step is about $10 \mathrm{~ms}$, which is much less than the update time of the Chiang algorithm (more than 200ms) ${ }^{[7]}$.

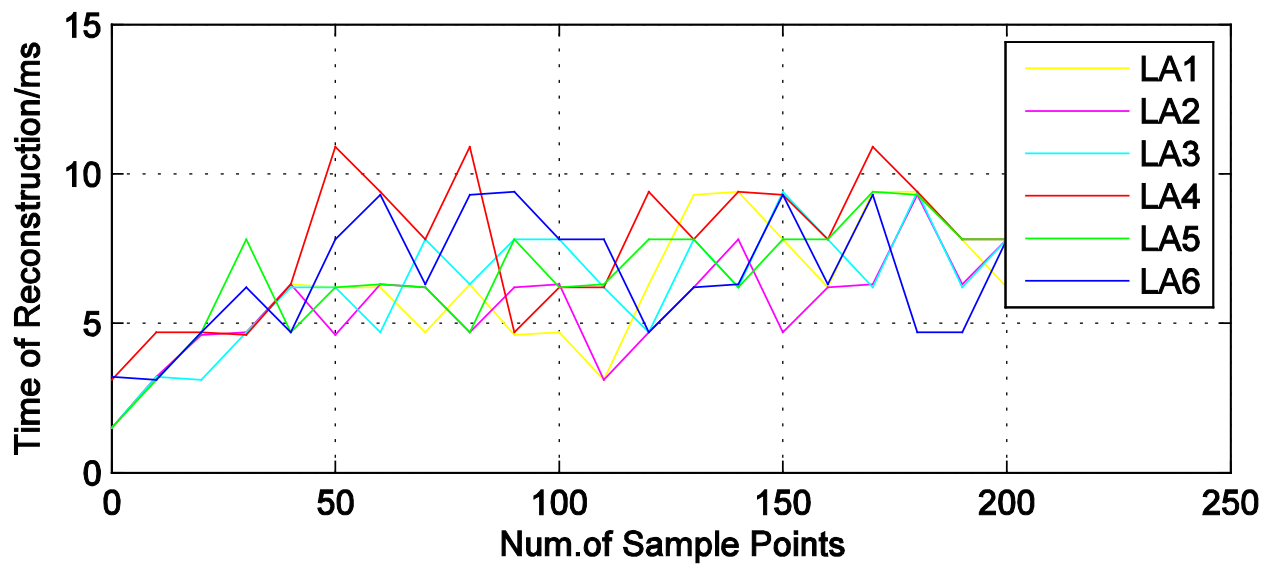

Fig. 8. Statistics of consumed time

\section{Conclusion}

The paper proposes a new method for dynamic reconstruction of heart chambers during ablation on the analysis of points acquisition process of the mapping catheter. The reconstruction results demonstrate that the method shows good performance by applying Delaunay diagram and the optimum surface constraints into dynamic surface reconstruction.

\section{References}

[1] Hoppe H, Derose T, Duchamp T, et al. Surface reconstruction from unorganized points[J]. Acm Siggraph Computer Graphics, 1999, 26(2):71-78.

[2] Thomas Lewiner, Hélio Lopes, Antônio Wilson Vieira, et al. Efficient Implementation of Marching Cubes' Cases with Topological Guarantees[J]. Journal of Graphics Tools, 2003, 8(2):1-15.

[3] Kazhdan M, Hoppe H. Screened poisson surface reconstruction[J]. Acm Transactions on Graphics, 2013, 32(3):61-70.

[4] Amenta N, Choi S, Kolluri R K. The power crust, unions of balls, and the medial axis transform[J]. Computational Geometry, 2000, 19(2-3):127-153.

[5] Peethambaran J, Muthuganapathy R. Reconstruction of water-tight surfaces through Delaunay 
sculpting[J]. Computer-Aided Design, 2014, 58:62-72.

[6] Reisfeld D. Three-dimensional reconstruction of intrabody organs: US, US 6456867 B2[P]. 2002.

[7] Chiang P, Zheng J, Mak K H, et al. Progressive surface reconstruction for heart mapping procedure[J]. Computer-Aided Design, 2012, 44(4):289-299.

[8] Loop C T. Smooth Subdivision Surfaces Based on Triangles[J]. Department of Mathematics the University of Utah Masters Thesis, 1987.

[9] M. Botsch, L. Kobbelt, M. Pauly, P. Alliez, B. Levy, Polygon Mesh Processing, AK Peters, 2010.

[10] Chaine R. A geometric convection approach of 3-D reconstruction[C]// EUROGRAPHICS SYMPOSIUM ON GEOMETRY PROCESSING (2003). 2003:218-229.

[11] Bowyer A. Computing Dirichlet Tessellations[J]. Computer Journal, 1981, 24(2):162-166.

[12] Watson D F. Computing the n-Dimensional Delaunay Tesselation with Application to Voronoi Polytopes[J]. Computer Journal, 1981,24(2):167-172. 